\title{
Wie Deutsche mit Ausländern sprechen und was man daraus für den Spracherwerb und den Sprach- unterricht lernen kann ${ }^{1}$
}

Jörg Matthias Roche, München

\section{$1 \quad$ Einleitung}

Darüber, wie Sprecher einer Zielsprache mit all denen kommunizieren, die diese Sprache nicht oder nur rudimentär beherrschen, wird sehr viel spekuliert. Obwohl die Sprachumgebung oder Eingabe im Spracherwerb anerkanntermaßen eine bedeutende Rolle spielt, gibt es erstaunlich wenige solide Untersuchungen des kom-

\footnotetext{
${ }^{1}$ Bei diesem Beitrag handelt es sich um den Plenarvortrag (gehalten am 9. Juni 2006) im Themenschwerpunkt 1 »Integrationskurse DaZ« im Rahmen der 34. Jahrestagung Deutsch als Fremdsprache des Fachverbandes Deutsch als Fremdsprache $(\mathrm{FaDaF})$ vom 8.-10. Juni 2006 an der Universität Hannover zum Rahmenthema »Chance Deutsch: Schule - Studium - Arbeitswelt«. Der Beitrag orientiert sich sehr stark am mündlichen Stil des Plenarvortrages und wurde nur leicht modifiziert. In einem solchen Vortrag ist es auch nur bedingt möglich, die relevante Forschungsliteratur angemessen darzustellen. So enthält dieser Beitrag naturgemäß einige Verkürzungen. Über die im Literaturverzeichnis angegebenen Referenzen kann der interessierte Leser dazu leicht Zugang finden.
} 
munikativen Verhaltens der Zielsprachensprecher in diesen ungleichen Kommunikationssituationen. Die Äußerungen der Lerner sind dagegen wesentlich besser untersucht. So kommt es, dass ein in Wirklichkeit recht komplexes, reiches, differenziertes und adaptives sprachliches Verhalten der Zielsprachensprecher hinter den vorwiegend indirekten, sporadischen und anekdotischen Beobachtungen versteckt bleibt. In diesem Beitrag möchte ich zunächst die authentische Sprache von Deutschen in der Kommunikation mit Lernern der Zweit- und Fremdsprache Deutsch präsentieren und analysieren. Sie wird dann im Kontext von Sprachentwicklungsprozessen mit anderen Formen und Registern reduzierter und grammatikalisierter Sprache verglichen. Abschließend wird ein Verfahren skizziert, das die systematische Integration reduzierter Sprachformen in den DaZ- und DaFUnterricht vorsieht. Dies erfordert jedoch eine Neudefinition des Progressionskonzeptes im Unterricht.

\section{Hypothesen über die Kommunikation mit Ausländern}

Wenn man sich ansieht, welche Hypothesen es zur Kommunikation mit Ausländern gibt, dann fallen am deutlichsten negative Einschätzungen auf. Es scheint wenig förderlich, wenn man mit Ausländern nicht korrekt redet, so die landläufige Vermutung. Der Begriff ,Stigmatisierung' erscheint häufig in solchen Kontexten. Eine andere Hypothese geht davon aus, dass eine mögliche gegenseitige Imitation von Lernern und Sprechern der Zielsprache den Spracherwerb verhindert. Wie soll ein Lerner schließlich auch die Sprache lernen, wenn beide rudimentäres Deutsch sprechen? Die nativistische Position zu dem Thema ist noch einfacher zusammengefasst: Der Input spielt dort naturgemäß eine sehr geringe Rolle. Schließlich wird davon ausgegangen, dass Lerner trotz unvollständigen Inputs die korrekte Grammatik entwickeln. Der Input ist also weder für den Spracherwerb noch für den Sprachunterricht relevant. Zu guter Letzt gibt es auch eine nicht sehr umfangreiche amerikanische Forschung zu diesem Bereich, die davon ausgeht, dass es keine besonderen Anpassungen der Zielsprachensprecher gibt, weil sie sie nicht beobachten konnte.

Vielleicht sagen Sie jetzt: Genau so ist es eigentlich, diese Stigmatisierung, dieses vermeintlich schlechte Deutsch, das wir mit Ausländern häufig reden, das kann ja nichts bringen. Ich möchte Ihnen dazu gleich eine kleine Begebenheit erzählen, die diese Annahme vielleicht bestätigt. Aber die Auflösung gebe ich Ihnen dann doch nicht gleich, denn die Geschichte nimmt noch eine überraschende Wendung. Es war also auf einer wichtigen großen internationalen Tagung, die interessanterweise noch auf Deutsch gehalten wurde, und in der Mittagspause saß ein kenianischer Konferenzteilnehmer beim Essen und ein deutscher Kollege setzte sich zu ihm, klopfte ihm dabei auf die Schulter und fragte: »Hamm hamm gut?« und man unterhielt sich ansonsten nicht, weil man sich mit 
vollem Mund bekanntlich nicht gut unterhalten kann. Schließlich prostete der Deutsche seinem Kollegen mit den Worten zu: »Gluck gluck gut?« .

\section{Struktur und Variation in der Kommunikation mit Ausländern}

So ungefähr stellen wir uns also die Kommunikation mit Ausländern vor. Aber in Wirklichkeit ist diese Sache viel differenzierter, und das möchte ich Ihnen nun anhand eines Transskripts zeigen. Dabei müssen Sie sich etwas an die Transkriptionsart gewöhnen. Die hat zwar phonetische Züge, kommt aber ohne phonetische Sonderzeichen aus und nutzt das graphemische System des Deutschen. Die Aufnahme stammt aus einem Korpus, das ich bereits vor einiger Zeit erstellt habe, und zwar mit Beobachtungen von authentischen Gesprächen zwischen Deutschen und Ausländern am Arbeitsplatz und in Geschäften und auch in privater Kommunikation. Wir haben also hier einen deutschen Sprecher D, nennen wir ihn mal Dietmar, und seinen türkischen Kollegen T, nennen wir ihn Tekin, die beide Mitte 20 sind, sich sehr gut kennen und befreundet sind. Und der Deutsche erzählt in dieser längeren Sequenz, was er am Vorabend gemacht hat ${ }^{2}$ :

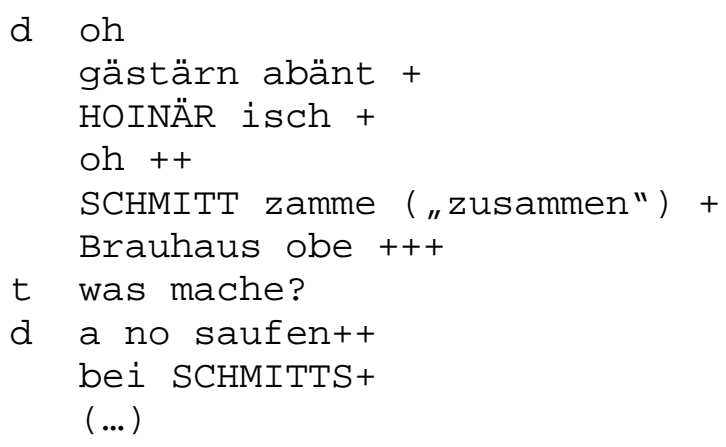

Natürlich kann man sagen, dass hier einige Funktionswörter, Endungen und andere Elemente fehlen. Es fehlt hier sicher mehr, als da ist. Man könnte dieses Gespräch als ungrammatisch beschreiben. Aber ich gehe davon aus, dass $90 \%$ von Ihnen schon verstanden haben, worum es hier geht, trotz der rudimentären Grammatik. Das ist auch mein Vorschlag, dass wir uns bei solchen Gesprächen nicht so sehr davon leiten lassen, was unserer Meinung nach falsch ist oder fehlt, sondern zunächst einmal sehen, was eigentlich da ist. So machen wir das im Übrigen ja mittlerweile auch in der Spracherwerbsforschung.

Die Struktur dieser Äußerungen, wie zum Beispiel »Gestern Abend Heiner isch«, lässt sich als fortlaufender Fokus beschreiben, d. h. als eine systematische Progres-

\footnotetext{
2 Zur Erläuterung der wichtigsten Zeichen: $\mathrm{c}=$ unbetontes $\mathrm{e}, \mathrm{x}=$ ach-Laut, $\mathrm{C}=$ ich-Laut, $B=$ schLaut, $\mathrm{z}=$ stimmhaftes $\mathrm{s},+=$ Pausenlänge (je circa 1 Sekunde), alle Namen sind geändert.
} 
sion von einem thematischen Element, in diesem Fall einem Temporaladverbial, zum Fokus. Und so geht das systematisch weiter. Der Fokus, die zusätzliche Information, die neue oder hervorgehobene Information, wird im Grunde einfach addiert. Hier ist ein weiteres Beispiel aus einer anderen Geschichte, das uns nachher noch etwas intensiver beschäftigen wird.

\begin{tabular}{|l|}
\hline där \\
\hline der
\end{tabular}$\rightarrow$\begin{tabular}{|c|}
\hline naxtßißt \\
\hline Nacht -
\end{tabular}$\rightarrow$\begin{tabular}{|l|}
\hline mäsär unt schärc \\
\hline Messer und Sche- \\
\hline
\end{tabular}

Thema

Fokus

Fokus

Sie können aus diesen wenigen Begriffen bereits ahnen, wie sich diese Geschichte weiterentwickelt. »Messer und Schere« ... und dann kommen die einzelnen Ereignisse. Hier liegt also die gleiche Struktur vor: Thema - Fokus und diese Struktur wird ergänzt durch den fortlaufenden Fokus. In der Literatur spricht man bei dieser Art Kommunikation von ,Foreigner Talk', ,Telegrammdeutsch' oder ,Ausländerdeutsch‘. Man könnte Neudeutsch auch ,SMS-Deutsch` sagen. Wegen der größeren Variation, die zu beobachten ist, und der Nähe zu anderen »-lekten« habe ich den Begriff ,Xenolekt' vorgeschlagen.

Sehen wir uns hier nun eine etwas komplexere Erzählung an und betrachten etwas genauer die Variation in dem Gespräch.

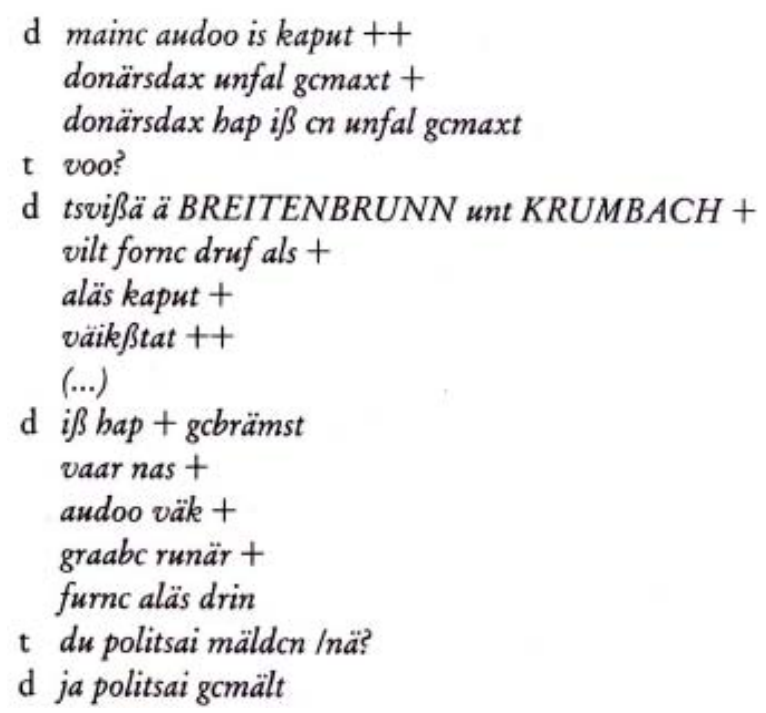

$\mathrm{d} i ß$ hap + gcbrämst vaarnas +

d ja politsai gcmält 


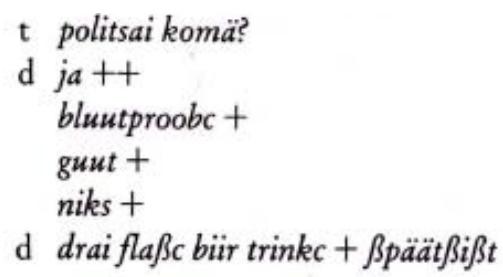

Sie erkennen natürlich auch den leichten dialektalen (hessischen) Einschlag. Den müssen wir dem Sprecher natürlich nachsehen. Hochdeutsch heißt es also:

»Mein Auto ist kaputt«.

»Donnerstag hab ich einen Unfall gemacht«.

$»$ Wo $\ll$ ? -

"Zwischen Breitenbrunn und Krumbach«.

»Wild vorne drauf. Alles kaputt - Werkstatt«.

»Ich hab’ gebremst, war nass. Auto weg. Graben runter. Vorne alles drin«.

$»$ Du Polizei melden«?

»a, Polizei gemeldet. Polizei gekommen. Ja. Blutprobe. Gut, nix. Drei Flaschen Bier trinken, Spätschicht«.

Das Interessante an diesem Gespräch ist außer dem Inhalt, seiner Darstellung und der überraschenden Pointe die Mischung der verschiedenen Äußerungstypen. Wenn man sich das Gespräch nämlich genauer ansieht, stellt man fest, dass einige der Äußerungen sehr rudimentär, infinitivartig und reduziert sind, d. h. dass Äußerungen nur mit Inhaltselementen auskommen. Es enthält aber auch Aussagen, die weniger verändert oder reduziert sind, wie zum Beispiel »Meine Auto ist kaputt", "Donnerstag hab' ich ein Unfall gemacht«. Einige sind sogar umgangssprachlich und enthalten eigentlich keinen Fehler. Und das ist eine ganz typische Sache, die wir noch ein bisschen weiter verfolgen sollten. Wir sehen gar keine registerartige Veränderung im Sinne eines Ausländerregisters, sondern einen enormen Variantenreichtum. Interessanterweise ist dieser Variantenreichtum bei vielen Sprechern zu beobachten, zumindest allen Sprechern, die ich untersucht habe, und die kamen aus ganz unterschiedlichen Gegenden und sozialen Schichten. Wir können also davon ausgehen, dass die hier beobachtete Variation kein Einzelfall ist, sondern systematisch auftritt. Wenn man sich jetzt den Textaufbau von solchen Erzählungen ansieht, dann stellt man fest, dass diese meistens nach dem folgenden Schema strukturiert sind:

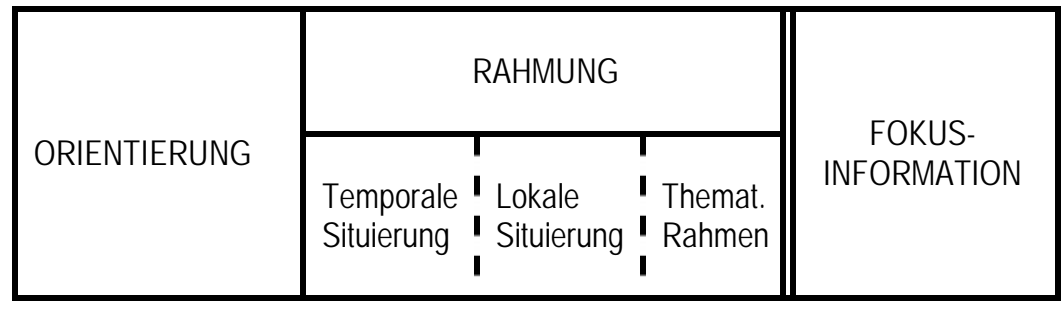


Es kann natürlich sein, wie in allen anderen Erzählungen auch, dass die eine oder andere Position nicht realisiert ist, aber im Grunde finden wir dieses Strukturmuster immer wieder: Eine Orientierung, wie in diesem Fall »Meine Auto ist kaputt«, die den Rahmen der Erzählung angibt, dann die temporale und thematische Situierung "Donnerstag hab ich ein Unfall gemacht«, in der normalerweise auch die handelnden Personen genannt werden. Im Grunde entspricht dieses Muster dem, was man in der Literaturwissenschaft eine ,Exposition' nennt, in der die wichtigsten Parameter am Anfang eingeführt werden.

Sehen wir uns nun noch einen weiteren Bereich an, nämlich den der semantischen Simplifizierungen. Zu beobachten ist in Xenolekten allgemein, dass häufiger eine Substitution durch Synonyme oder vom Sprecher als Synonym erachtete Begriffe - ich nenne sie ,Pseudosynonyme - und Paraphrasen erfolgt. Also statt »Leben« wird »Wohnen« gesagt, statt »ärgern« »aufregen«, wahrscheinlich wegen der Ausdifferenzierung, die durch das Präfix erfolgen kann. Statt »überprüfen« wird »Kontrolle« verwendet, möglicherweise, weil »Kontrolle« als Internationalismus vermutet oder erkannt wird. 1500 wird aufgeteilt in »1000 - 500«, »jedes Mal« wird zu »immer«. Zudem gibt es auch einige Neologismen, die spontan geschaffen werden, wie zum Beispiel anstatt »einen Kredit aufnehmen« »über Kredit gehen« oder anstatt »Schleudertouren« »Schleuderzahl«, weil der Sprecher »Zahk offensichtlich als prägnanter erachtet. Zusätzlich scheinen metonymische Kürzungen ein beliebtes Mittel zu sein: »Möbelabteilung« erscheint als »Möbel«, »Teppichabteilung« als »Teppiche«. Auf der anderen Seite werden semantische Elemente analytisch präsentiert wie in »große Baum mit Blätter« statt »Eiche«, »viel Reparatur« statt »reparaturanfällig« (Quantifizierung), »meist gängige« statt »das gängigste« (Komparation) und »Monat, Monat« statt »monatlich" (Iteration). Solche analytischen Wiederholungsformen sind übrigens auch ein beliebtes Mittel von Lernern im Zweitsprachenerwerb, um wiederholte oder anhaltende Handlungen auszudrücken.

\section{Sprecher- und adressatenspezifische Variation}

Kommen wir nun zu dem Thema ,Variation unter den Sprechern und adressatenspezifische Variation'. Ich habe bisher versucht, das System der Xenolekte darzustellen, aber man könnte sich ja im Anschluss daran fragen, wie einheitlich diese Kommunikation eigentlich ist. Reden wir relativ pauschal mit Ausländern, spielt der Ausländerstatus, die Erscheinungsweise des Adressaten eine Rolle, passen wir uns an das Sprachniveau an und gibt es individuelle, sprechertypische Präferenzen? Dazu möchte ich Ihnen aus meinem Korpus exemplarisch nur einen Sprecher vorstellen und kurz erklären, was diese Abbildung bedeutet (aus: Roche 1989: 148). 


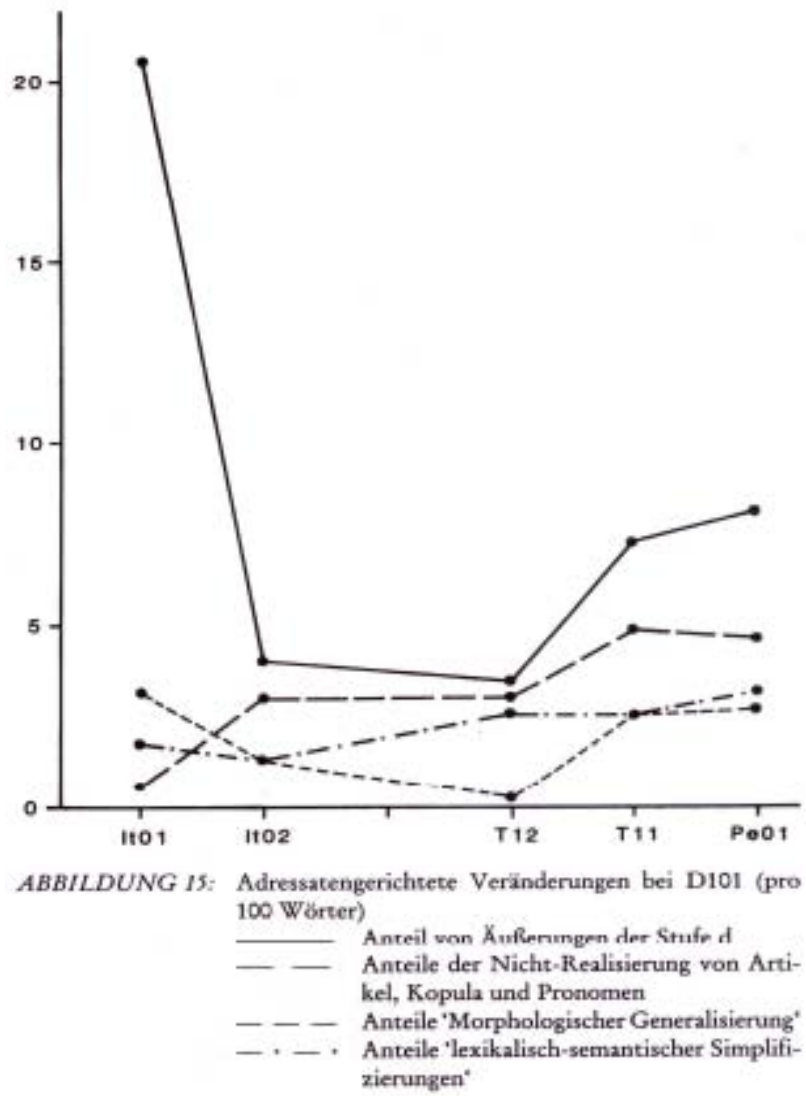

Auf der vertikalen Achse ist die Vorkommenshäufigkeit und auf der horizontalen Achse sind die verschiedenen Adressaten abgetragen. IT01 ist ein italienischer Gastarbeiter, der wirklich sehr schlecht Deutsch spricht, obwohl er zum Zeitpunkt der Aufnahme 15-20 Jahre in Deutschland gelebt hat. IT02 ist auch ein italienischer Gastarbeiter, der aber über etwas bessere Kenntnisse verfügt. T12 ist ein türkischer Gastarbeiter, T11 ein türkischer Krankenpfleger und PE01 ein peruanischer Student mit sehr guten Deutschkenntnissen. Die Sprachkenntnisse dieser Versuchspersonen, die ich hier nur grob beschrieben habe, habe ich natürlich in einem Blindversuch von einer Kontrollgruppe bewerten lassen. Dazu habe ich Sprachausschnitte aus den Aufnahmen genommen, habe sie der Kontrollgruppe vorgestellt und habe sie in Bezug auf die Verständlichkeit bewerten lassen. Daraus ergibt sich diese Reihung, wobei die Abstände zwischen den Sprechern nicht maßstabsgetreu sind. Aus der Bewertung ergibt sich immerhin eine signifikante Zunahme der Verständlichkeit nach rechts. Der Peruaner spricht fast muttersprachlich Deutsch. IT01 kann tatsächlich nur ein paar ganz rudimentäre Brocken Deutsch. 
Anhand von verschiedenen Merkmalen kann damit gemessen werden, wie sich ein deutscher Verkäufer in einem großen Kaufhaus, der täglich mit unzähligen Kunden zu tun hat, in einer Standardsituation verhält. Die Standardsituation bestand hier aus dem Waschmaschinenverkauf, Garantieanfragen, Reparaturfragen und Ähnlichem. Alle meine Versuchspersonen hatten dabei die Aufgabe, die gleichen Fragen an diese Person zu stellen. Allerdings geschah dies mit einem Abstand von mehreren Tagen oder Wochen, so dass der Verkäufer keinen Verdacht schöpfen konnte.

Die durchgezogene Linie bildet die Infinitiväußerungen $\mathrm{ab}$, das sind diese rudimentären Äußerungen, die wir anfangs gesehen haben. Es zeigt sich dabei, dass der Verkäufer gegenüber dem sehr schlecht Deutsch sprechenden und verstehenden Italiener eine ganz hohe Anzahl der rudimentären Äußerungen benutzt. Circa die Hälfte aller Äußerungen des deutschen Verkäufers sind solche Infinitiväußerungen. Das fällt dann rapide ab. Die anderen Strategien, wie die Nicht-Realisierung von Artikel, Kopula und Pronomen, verwendet er viel seltener. Morphologische Generalisierungen, damit sind vor allem Flexionsausfälle und -reduktionen gemeint, verwendet er bei allen Adressaten, aber relativ wenig, und lexikalischsemantische Simplifizierungen benutzt er nur gelegentlich. Aber das Auffällige hieran ist, dass er seine Sprache sehr stark an den jeweiligen Adressaten anpasst. Gegenüber dem Italiener IT01 erwarten wir das. Dass die starken Anpassungen so rapide abfallen, ist aber nicht unmittelbar verständlich. Dass die starken Veränderungen bei den besser sprechenden Adressaten wieder ansteigen, scheint auf den ersten Blick vielleicht sogar paradox. Diese Adressaten sprechen eigentlich ganz gut Deutsch, also müsste man annehmen, dass der Sprecher mit ihnen auch ganz normales Deutsch spricht. Woran liegt das? Sehen wir uns dazu noch eine weitere Folie an. Sie illustriert, wie verschiedene Sprecher sich gegenüber den gleichen Versuchspersonen verhalten. Hier habe ich mittels eines Verfahrens Typen eines Wortes und Tokens, also ihre Häufigkeit, gemessen. Es geht um die Inhaltsdichte des Gesprächs anhand des Vorkommens von Wörtern und ihrer Häufigkeit (vgl. Roche 1989: 119). 


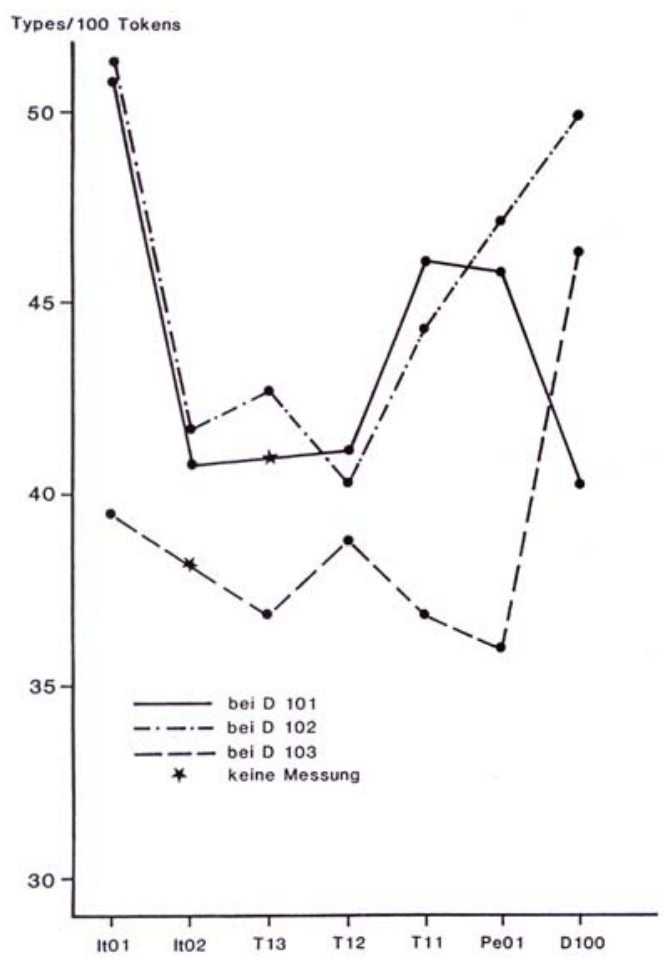

ABBILDUNG 13: Type-Token-Verhältnis in den Auskunftsdaten

Hier sehen Sie die Werte von drei Versuchspersonen: D101, einem anderen Verkäufer D102, der Fernsehgeräte verkauft, und D103, der in einer Lotterieannahmestelle arbeitet. Und Sie sehen schon, ohne dass ich auf die Einzelheiten eingehe, dass es große Unterschiede zwischen den Sprechern gibt. Beachten Sie bitte, die Informanten reden immer mit den gleichen Adressaten, die Thematik ist die gleiche und dennoch haben sie andere Präferenzen. Bei D101 und D102 sehen wir eine gewisse Übereinstimmung, aber gegenüber dem Peruaner und einer muttersprachlichen Vergleichsperson (D100) zeigen sich doch ganz andere Strategien. Hier ist das Verhältnis von Wörtern und Vorkommen relativ hoch, d. h., es liegt eine sehr hohe Inhaltsdichte vor. Fast jedes Wort kommt maximal zweimal vor, während gegenüber den weiteren Adressaten das Type-Token-Verhältnis niedriger ist. Das bedeutet, die Sprecher verwenden gegenüber IT01 sehr viele Inhaltswörter und lassen die Funktionselemente weg, die ja im Deutschen hochfrequent sind. Der erneute Anstieg bei den Adressaten rechts lässt sich auf qualitative Unterschiede zurückführen. Gegenüber den schlecht sprechenden Adressaten beschränken sich die Sprecher auf einfachste Erklärungen, z. B. zum Funktionieren der 
Maschine, des Fernsehers oder des Lottosystems, und auf ein paar kurze Informationen. Schluss. Die fortgeschrittenen Adressaten sind dagegen viel flexibler, die können nachfragen, die können Zusatzinformationen verlangen. Dementsprechend geben die deutschen Sprecher nicht nur kurze Erklärungen. Sie reagieren auf die Nachfragen und geben entsprechende Nachlieferungen, und die sind teilweise sehr komprimiert, aber dann auch wieder ausgedehnt. Das erklärt, warum sich das Type-Token-Verhältnis auch bei besseren Adressaten erhöht. Das Gespräch von D101 und der deutschen Versuchsperson, der letzte Punkt in der Abbildung, findet ganz im Dialekt, ganz umgangssprachlich, statt. Es handelt sich um ein sehr facettenreiches Gespräch, aber es verläuft in den Routinen der Umgangssprache, und die sind häufig unterspezifiziert. Da heißt es anstatt »Waschmaschine« zum Beispiel einfach »Ding«, weil man sich auf eine gemeinsame Basis beziehen kann, also können die Wörter und Strukturen auch unterspezifiziert und elliptisch sein. Wir sehen hier, dass es sowohl eine sprecherspezifische Varianz für verschiedene Strategien gibt und eine gewisse adressatenspezifische Anpassung. Als Sprecher der Zielsprache scheren wir also nicht alle ausländischen Adressaten über einen Kamm, wie das häufig vermutet wird. Xenolekte sind kein einheitliches Register.

Aber es geht noch weiter. Sehen wir uns nun das, was wir gerade quantifiziert betrachtet haben, noch einmal unter qualitativen Gesichtspunkten genau an. Dieses Gesprächssegment stammt aus einem etwa 30-40minütigen Gespräch zwischen einem deutschen und einem türkischen Kollegen. Dabei erzählt der Deutsche von verschiedenen Sachen, von einem Unfall, von einer Messerattacke und Ähnlichem. Eine Beispieläußerung haben wir vorhin schon gesehen. Sehen wir mal, wie seine Sprache variiert:

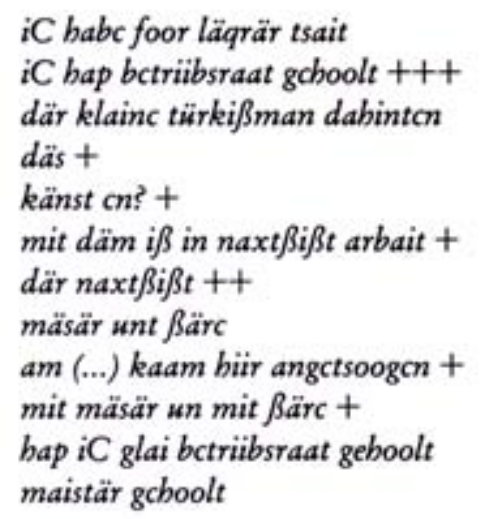

»Ich habe vor einer längeren Zeit, der kleine Türkischmann da hinten«, diese ÄuBerung entspricht im Grunde einer umgangssprachlichen Äußerung mit der Ausnahme von »Türkischmann« als semantischer Simplifizierung. Grammatisch ist diese Äußerung korrekt. Dann gibt es aber auch eine Reihe ganz stark veränderter 
Äußerungen, also infinitivartige, rudimentäre Äußerungen. Es finden sich aber weitere rein umgangssprachliche Äußerungen wie diese Nachfrage »kennst ihn« und diese Äußerung »kam hier angezogen«. Zudem gibt es Äußerungen, die in einem Element verändert sind, wie zum Beispiel »ich hab' Betriebsrat geholt« statt wich hab' den... « oder »mit dem ich in Nachtschicht Arbeit» anstatt »mit dem ich in der ...«. Es fehlt hier also der Artikel. Es ist typisch für diese Art von Äußerungen, und auch über Sprechergrenzen hinweg, dass immer nur ein Element von Auslassungen betroffen ist. Es fehlt entweder eine Präposition oder ein Artikel, aber es fehlt in diesem Äußerungstypus in ganz seltenen Fällen beides. Das Inventar der Sprecher besteht demnach aus vier Äußerungstypen:

- umgangssprachlichen Äußerungen,

- semantisch vereinfachten oder verlangsamten Äußerungen,

- in einem Element betroffenen Äußerungen oder

- infinitivartigen Äußerungen.

\section{$5 \quad$ Pragmatische Variation}

Der deutsche Sprecher erzählt von der Messerattacke und rekurriert dabei auf einen türkischen Kollegen. Den realisiert er in seinen Erzählvarianten unter anderem als »Türkischmann«, »des«, »««, »er«, »den«, »der«, mit seinem Vornamen und auch als Ellipse (»ich kann dir zeigen«). Diese Variantenvielfalt gilt für alle der beobachteten Sprecher und betrifft nicht nur die Lexik, sondern, wie wir schon gesehen haben, auch die Grammatik. Wenn man sich den Codewechsel genauer ansieht, dann kann man feststellen, dass es mindestens sechs verschiedene Veränderungsniveaus gibt, die jeweils spezifische Funktionen markieren: Ein

- Ausgangsniveau

- Erklärungs- und Erzählniveau

- Komprimierungsniveau

- Zitatniveau

- Nachlieferungs- und Nebenthemenniveau

- Kommentarniveau

Das Ausgangsniveau bildet eine erste augenscheinliche Einschätzung ab. Darunter fallen auch Kriterien wie Hautfarbe und weitere sprachliche und außersprachliche Merkmale der »Ausländerhaftigkeit«, die wir rational gar nicht immer erklären können. Ich nenne es ,Ausgangsniveau', weil es sehr instabil ist. Nach meiner Beobachtung versuchen die Sprecher, schnell von diesem Niveau wegzukommen. Sie versuchen eigentlich nur zu reduzieren und einfacher zu sprechen, wo es unbedingt nötig ist. Dabei sollte man berücksichtigen, dass ein reduziertes Sprechen eigentlich sehr viele Kapazitäten verlangt, weil es von unserer automatisierten Gesprächsroutine massiv abweicht. Als zweites Niveau lässt sich das Erklä- 
rungs- und Erzählniveau bestimmen. Es betrifft die zentralen, inhaltlichen Elemente, also die zentrale Bedeutungsvermittlung (Proposition). Die Erklärungsund Erzählsequenzen sind in der Regel am stärksten verändert oder reduziert. Wo die inhaltliche Vermittlung, der propositionale Gehalt, uns Sprechern ganz besonders wichtig ist, da reduzieren wir, da konzentrieren wir uns, da verwenden wir die 1-Wort-Äußerungen und die infinitivartigen Sätze. Ganz ähnlich betrifft das Komprimierungsniveau kurzfristige Erklärungen als Antwort auf Nachfragen, etwa nach den Schleudertouren einer Waschmaschine. Dann wird temporär mit einzelnen Begriffen komprimiert und vertieft, aber sofort wieder in ein anderes Niveau gewechselt.

Das Zitatniveau ist ebenfalls ganz interessant, weil es eine Strategie repräsentiert, die wir automatisch beherrschen, um uns zu helfen. Es lässt sich beobachten, dass Sprecher, die irgendetwas erzählen oder erklären, gerne auf originale Referenzen Bezug nehmen und original zitieren. Sie übernehmen Zitate von Dritten eins zu eins und betten diese auch in komprimierte Kontexte ein.

Zudem gibt es so etwas wie ein Nachlieferungs-/Nebenthemenniveau, das in der Regel viel weniger reduziert oder angepasst wird, als die anderen Äußerungen. Wenn ein Sachverhalt oder Begriff erklärt wurde und den Sprechern auffällt, dass das eine oder andere Element noch erklärt werden muss, dann wird diese Information im Sinne von »ich meine .... nachgeliefert. Äußerungen werden dann viel weniger reduziert als bei dem Erzähl- und Erklärungsniveau. Schließlich gibt es noch das Kommentarniveau, das an die Codewechsel in Luthers Tischreden erinnert. Sprecher nutzen es ohne erkennbaren Zeitverlust, um Textpassagen oder Sachverhalte zu kommentieren, und wechseln dabei zum Beispiel von einer stark komprimierten Äußerung in ihren Dialekt und wieder zurück, so wie Luther vom Lateinischen ins Deutsche wechselte, wenn er seine Reden kommentierte oder schimpfte. Wenn zum Beispiel D101 das Funktionieren einer Waschmaschine erklärt und illustriert und die Tür geht in diesem Moment gerade nicht zu oder auf, dann fängt er an zu fluchen. Das tut er aber nicht in den xenolektalen Äußerungen, sondern da wechselt er sofort in die Begrifflichkeit seines Dialektes, wie gesagt nahtlos. Der Kunde ist in solchen Fällen nicht der primäre Adressat.

Kann man nun sagen, wie es ja häufig gemacht wird, diese Kommunikation drückt vor allem die Abwertung der Ausländer, eine Stigmatisierung, aus? Fragen wir hier stellvertretend Dietmar, von dem die vorhin besprochene Erzählung stammt, wie er das sieht, trotz der Messerattacke. Er sagt:

ich hab' nix gegen türkischmann, türkischfrau

hab ich nimmer

nur ihr anders sprechen wie wir ne?

anders sprechen

andre sprache

ich hab nix dagegen 
Es geht bei Xenolekten also mit Sicherheit primär nicht um die Stigmatisierung oder Abwertung der Ausländer/Lerner, sondern es geht vor allem um die Aufrechterhaltung der Kommunikation. Die Art und Weise mit Ausländern zu reden ist ein produktives und nicht ein abwertendes Mittel. Die meisten meiner Informanten empfinden diese Anpassungen als große Hilfe. Einige haben dies jedoch eingeschränkt, nämlich diejenigen, die schon besser Deutsch konnten. Sie wollen verständlicherweise nicht mehr so angesprochen werden, wenn sie schon Deutsch können. Aber auch sie haben bestätigt, dass Komprimierungen eine groBe Hilfe für sie darstellten, als sie noch nicht so gut Deutsch sprachen, denn so konnten sie überhaupt etwas verstehen. Das ist ein interessanter Befund, der so gar nicht in die Landschaft vorgefertigter Meinungen passt.

\title{
6 Englischsprachige Xenolekte
}

Noch ein interessanter Befund: in der englischsprachigen Literatur wird oft behauptet, dass es solche Xenolekte gar nicht gebe, und wenn, dann sei das eine deutsche Eigenart. Zu dieser Annahme habe ich vor einigen Jahren mit Unterstützung der Alexander-von-Humboldt-Stiftung in Kanada eine kontrastive Studie durchgeführt.

\author{
Explaining 'a simpler washing machine' \\ Salesman: well this + \\ here is a simpler ++ \\ this is a very simple + \\ okay? ++ \\ this very basic \\ is just all normal + \\ no delicate \\ no permanent press + \\ okay? \\ is just very basic + \\ you go from here + \\ and then when you go to this one here + \\ see \\ this one has a three speeds + \\ and a washer \\ its watertemperature + \\ but that one you can't do much with is \\ ES: okay is much simpler \\ Salesman: yeah +++
}


In diesen wenigen Äußerungen des Verkäufers (in einem großen Warenhaus in Toronto) finden sich einige Parallelen zu den deutschen Gesprächen. Typisch für Sprecher in beiden Sprachen ist dabei, welche Probleme sie haben, das richtige Niveau zu finden. Diese Schwierigkeiten manifestieren sich in den vielen Abbrüchen am Anfang des Gesprächs. Der Sprecher entwickelt hier hörbar einen Plan und während er den Plan entwickelt, bemerkt er, dass sein Gegenüber das vermutlich gar nicht verstehen wird, weil er es auf Grund der Hautfarbe vermutet, weil die Kundin aus Ecuador eine ungewöhnliche oder spezifische Gestik oder Mimik an den Tag legt oder weil irgendwelche anderen Anzeichen vorhanden sind, die Nicht-Verstehen oder Verstehensprobleme signalisieren. Daraus resultieren die vielen Ansätze, bis dann das passende Niveau ausgetestet ist.

Ich habe in Kanada auch Zugang zu einem bedeutenden Archiv des Ontario Folklife Centres gehabt, für das Kolleginnen und Kollegen Interviews mit Immigranten durchgeführt hatten, die in den 50er Jahren nach Kanada gekommen waren. Aus diesem Korpus zeige ich Ihnen hier einen Ausschnitt aus einem langen Gespräch zwischen dem englischsprachigen Interviewer Marc (MF) und einem älteren Informanten aus Litauen (GZ). Der litauische Informant fragt nun auch den Interviewer ein paar Sachen:

\section{Mennonite}

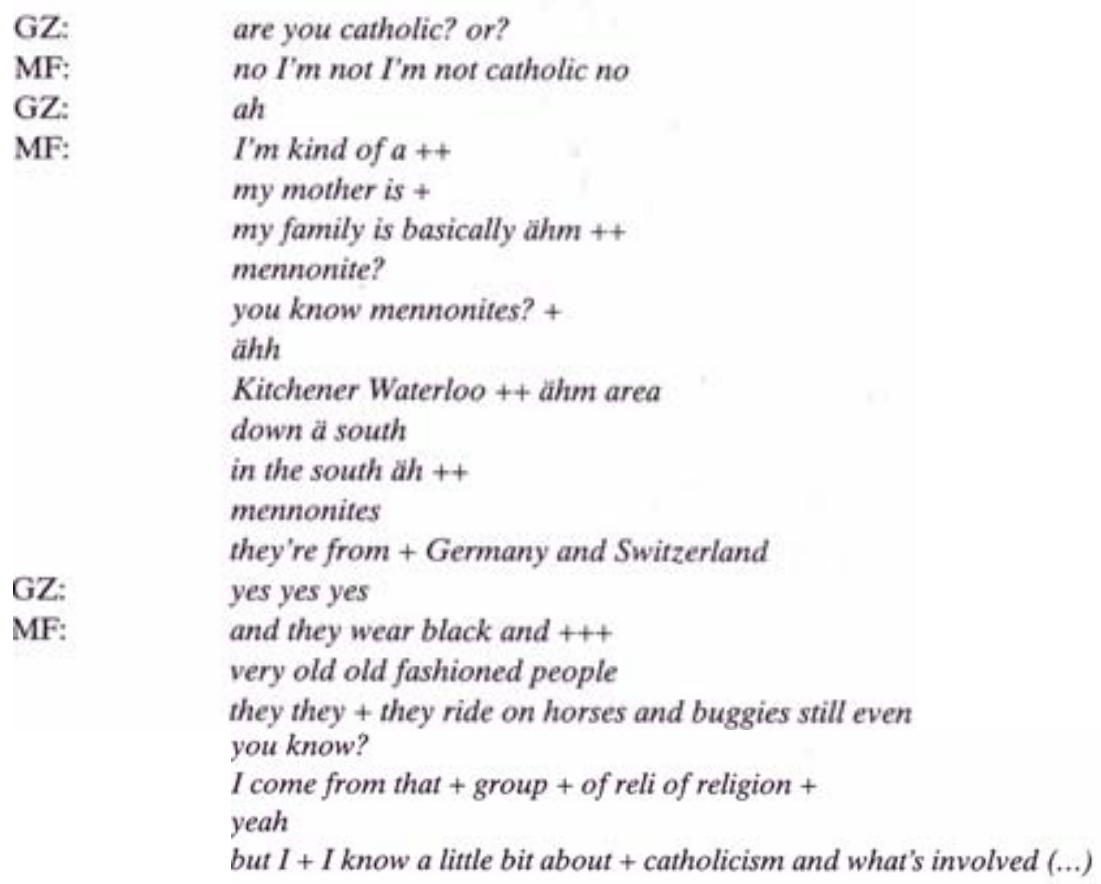


Dazu müssen Sie wissen, dass die genannten Orte ,Kitchener ${ }^{6}$ und ,Waterloo“ Ansiedlungen südlich von Toronto sind, in denen viele Menonniten leben. Der Interviewer versucht hier mit einer metonymischen Ortsangabe, ein kulturelles und religiöses Konzept zu erklären, und all das in sehr rudimentären Äußerungen. Also auch dieses Stereotyp, dass Xenolekte in dieser Ausprägung typisch für das Deutsche sind, können wir beiseite legen. Xenolekte gibt es auch im Englischen. Für das Türkische hatte sie bekanntlich Volker Hinnenkamp (vgl. Hinnenkamp 1982) bereits belegt. Die Akzeptanznormen sind aber in verschiedenen Kulturen unterschiedlich.

Wenn man etwa einen Kanadier mit einer solchen Aufnahme konfrontiert, was ich bei MF gemacht habe, dann läuft er rot an und es ist ihm sehr peinlich. Das erklärt, dass bei amerikanischen Untersuchungen bisher wenige verlässliche Daten produziert wurden. Hier wurden nämlich zum Großteil Informanten aufgenommen, die im Geschäftsbereich öffentlich tätig sind, und da redet man natürlich nicht so rudimentär wie am Arbeitsplatz, und schon gar nicht, wenn ein Mikrofon eingeschaltet ist.

\section{$7 \quad$ Pragmatischer und syntaktischer Modus}

Zum Abschluss möchte ich das skizzierte System in einen sprachgenetischen Kontext einbetten, wie ihn Givòn schon 1979 vorgestellt und später weiter entwickelt hat. Sein Modell beschreibt sowohl die phylogenetische als auch die ontogenetische Sprachentwicklung als ein Wechselspiel von einem pragmatischen und einem syntaktischen Modus. Alle die Elemente, die wir in den Äußerungen der Xenolektsprecher gesehen haben, eine klare syntaktische Struktur, langsamere Sprechgeschwindigkeit, pragmatische Prinzipien der Informationsverteilung, ein insgesamt hohes Verhältnis von Verben und Nomen - also den wichtigsten Inhaltswörtern -, wenig grammatische Morphologie und ganz bestimmte Intonationsmuster, sind typische Merkmale des pragmatischen Modus. Der syntaktische Modus dagegen beschreibt den Zustand der Grammatikalisierung, wie sie etwa in der deutschen Hochsprache ausgeprägt ist. Allerdings zeigen uns die Tendenzen der deutschen Gegenwartssprache genau das Wechselspiel zwischen pragmatischem und syntaktischem Modus: Ist der syntaktische Modus voll entwickelt, setzen Verschleifungserscheinungen ein. Man sieht das im Gegenwartsdeutsch unter anderem

- an der Reduktion des Kasusinventars;

- an der Tendenz zu analytischen statt synthetischen Formen, etwa beim Konjunktiv mit ,würde', dem Präteritumschwund oder dem Verschwinden starker Verbformen (,backte' statt ,buk');

- an der Hauptsatzstellung bei ,weil'; 
- an der Reduktion der Flexionsmorphologie in der gesprochenen Sprache; an der Verkürzung der Klammerstrukturen zugunsten von Ausklammerungen, Nachträgen und Ähnlichem.

Das Englische ist uns hier bekanntlich schon etwas weiter voraus. Das, was Givòn als Modi beschreibt, lässt sich aus Lernersicht etwa folgendermaßen darstellen (Roche 2005: 101):

\section{Prinzipien des Spracherwerbs}

- Bekannte und gegebene Information steht vor neuer Information.

- Thematisierende Elemente stehen vor fokussierenden Elementen.

- Bedeutungsmäßig zusammengehörige Elemente stehen möglichst nahe beieinander.

- In einer Reihung von Nomen hat das erste Element den größten Einfluss.

- Funktionale Elemente wie kein, viel, alle werden einheitlich vor (oder einheitich hinter) die von ihnen bestimmten Elemente gestelt.

- Orientierende Elemente wie Orts- oder Zeitangaben stehen am Anfang einer Außerung.

- Ereignisse werden nach inrer tatsächlichen (chronologischen) Reihenfolge berichtet.

- Die Betonung bestimmt, ob es sich um eine Aussage, eine Frage oder eine Anweisung handelt.

- Die Betonung markiert auch die fokussierten Elemente.

Die Prinzipien des Lerners zeigen dabei eine frappierende Übereinstimmung mit den Vereinfachungsstrategien der Xenolektsprecher. Daraus könnte man schließen, dass sich Lerner und Zielsprachensprecher gegenseitig so imitieren, dass am Ende ein gemeinsames Pidgin herauskommt. Wir haben aber bereits in der Variation der Xenolekte gesehen, dass dies nicht der Fall ist. Eingabe ist nicht gleich Ausgabe. Vielmehr treffen die Lerner bekanntlich auf der Basis ihrer Vorkenntnisse und Annahmen eine bestimmte Auswahl (Aufnahme) und verarbeiten diese nach sequenztypischen, sprachtypischen und individuellen Möglichkeiten. Daraus entsteht dann die Lernerproduktion (Ausgabe).

\section{Konsequenzen für den Sprachunterricht}

Fassen wir also kurz den Zwischenstand zusammen:

1. Die Eingabe bietet ein reichhaltiges Inventar.

2. Sie wird individuell variiert und enthält viele Paraphrasen.

3. Die Variation ergibt sich oft aus expliziten Aushandlungsprozessen. 
4. Die Variation ist keine registerartige pauschale (auch wenn pauschalierende Einschätzungen der Adressatenkompetenz besonders am Anfang von Kontakten vorkommen), sie ist pragmatisch bestimmt, nach der Art der Illokution und nach dem Gewicht des propositionalen Gehalts.

5. Der Anpassungsaufwand für Sprecher ist hoch. Sie versuchen daher so schnell wie möglich zu ihrer »normalen« Sprache zurückzukehren.

6. Xenolekte gibt es nicht nur im Deutschen.

Aus der Sicht des Lerners ergibt sich daraus:

1. ein höchst vielfältiger Input;

2. die interaktive Aushandlung verstehbaren Inputs und die Abstimmung von Erwerbsstand und Eingabe (allerdings nicht im naiven Sinne von Krashens Inputtheorie) (vgl. Krashen 1985);

3. eine Hilfsfunktion der Xenolekte statt einer Stigmatisierungsintention.

Aus diesen Beobachtungen lassen sich meines Erachtens daher einige brauchbare Folgerungen für die Erprobung im Unterricht ableiten:

1. Die Art und Weise, wie die Eingabe ausgehandelt wird. Es sollte grundsätzlich viel direkter an das Niveau der Lerner angeknüpft als einer vorgeschriebenen, von der Forschung nicht abgesicherten grammatischen Progression gefolgt werden. Dazu muss man sich auch undogmatischer Mittel bedienen, und zwar nicht nur im lexikalischen Bereich (etwa durch die Verwendung von Internationalismen), sondern auch im grammatischen.

2. Die Progression bei produktiven und rezeptiven Fertigkeiten kann und sollte konsequent anders gestaltet werden. Nicht alles, was ein Lerner verstehen kann, kann er auch sofort umsetzen.

3. In den Progressionen ist eine angemessene Nutzung von Textsorten im Sinne der Abstimmung auf die Verarbeitungsmöglichkeiten der Lerner und die natürlichen Anpassungsstrategien der Zielsprachensprecher angeraten.

Welche Textsorten dafür in Frage kommen, will ich an folgenden Beispielen illustrieren. 


\section{rasen}

\section{tötet}

freunde kinder tante bekannte

kumpel opa schwester oma mutter

arbeitskollegin partner bruder

ehefrau vater opa neffe oma

freundin onkel lebensgefährtin

kind mutter opa bekannte oma

ehemann neffe $\mathbf{b e k a n n t e}$ kind

onkel mutter tante vater oma

freund bekannte nichte freunde

kind tante bekannte kumpel

schwester mutter arbeitskollegin

partner bruder ehefrau vater opa oma

freunde freundin onkel bruder

freund bekannte nichte freunde

kind tante onkel neffe vater mutter

ehefrau freun

lebensgefährt

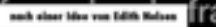

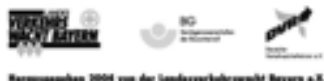

Dieser Text der Verkehrswacht, der lange an vielen Autobahnen zu lesen war, enthält außer der 2-Wort-Äußerung des Titels nur einzelne Wörter und eignet sich damit sehr gut für den Aufbau eines Wortfeldes in den ersten lexikalischen Phasen des Spracherwerbs. Lehrwerke, wie Schritte international (2005), setzen diesen Ansatz bereits folgendermaßen um, wobei durchaus auch einzelne Wortketten (Phrasen, »Chunks«) vorkommen können, die allerdings nicht grammatisch analysiert werden brauchen.

2 B3 Ratsel: Wer bin ich? Sprechen Sie mit Ihrer Partnerin / Ihrem Partner.

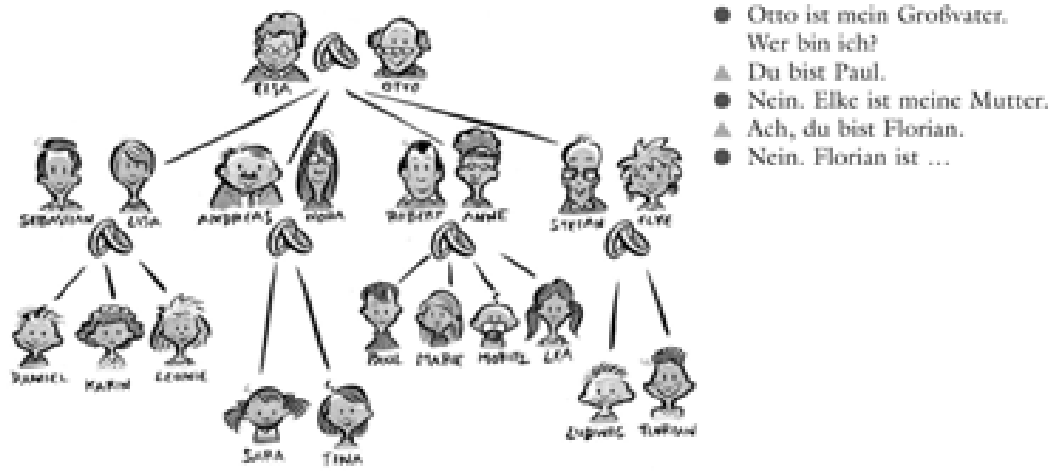


In den folgenden beiden kombinierten Textsorten finden Sie zudem weitere völlig authentische Äußerungsstrukturen (Sätze, Texte), die allerdings nach pragmatischen Prinzipien gebildet werden, also einfach gesagt, ohne Grammatik auskommen und dennoch völlig korrektes Deutsch darstellen.

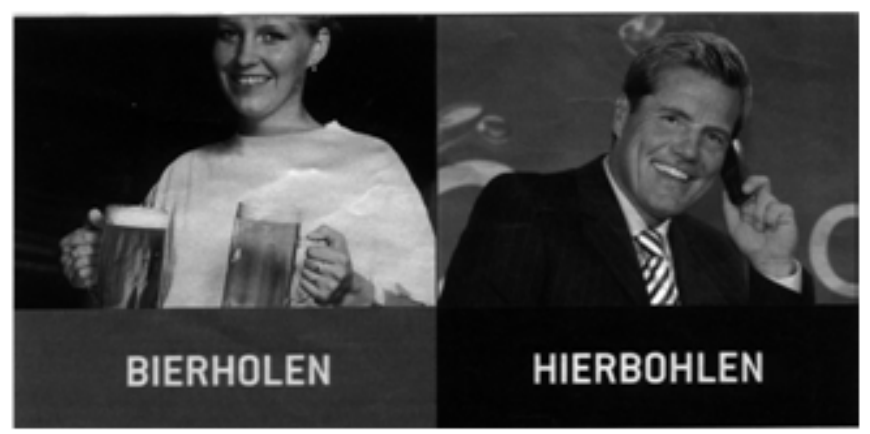

In den beiden folgenden Beispielen aus Tangram (2001) und Schritte international (Band 1, 2005) sehen Sie, wie diese pragmatischen Strukturen produktiv (und motivierend) für das Sprachverstehen und die Sprachproduktion bei Anfängern eingesetzt werden können. Die Lerner können so viel schneller als durch zu frühe und zu intensive Grammatikalisierung kommunikative Flexibilität erlangen.

Beispiel: Verwendung einfacher Strukturen im Unterricht (aus Tangram und Schritte International 1):

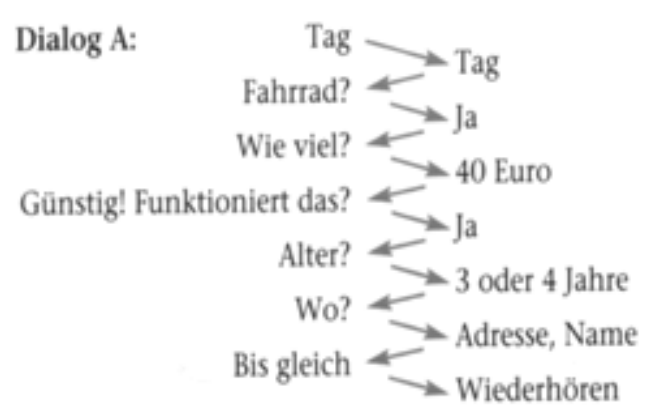


D4 Hören Sie das Telefongespräch und sprechen Sie dann mit Ihrem Namen.

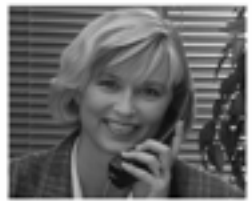

Firma Teletec, Iris Pfeil, guten Tag.

Guten Tag, Herr K...

Entschuldigung, wie ist thr Name?

Ah ja, Herr Khosa. Tut mir Leid, Frau Söll ist nicht da.

Auf Wiederhoren, Herr Khosa.

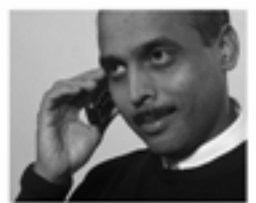

Guten Tag. Mein Name ist Khosa. Ist Frau Soll da, bitte?

Khosa.

Khosa. Ich buchstabiere: $\mathrm{K}-\mathrm{H}-\mathrm{O}-\mathrm{S}-\mathrm{A}$.

Ja, gut. Danke. Auf Wiederhören.

An dem folgenden bekannten authentischen Text kann man leicht erkennen, wie facettenreich Wortschatz mit einfachen grammatischen Strukturen genutzt werden und damit sukzessive zum Aufbau einer grammatischen Kompetenz beitragen kann. Nach der lexikalischen Phase geht es also um die Nutzung einfacher grammatischer Strukturen wie hier der prädikativen Nutzung der Adjektive statt der der flexionsreicheren attributiven.

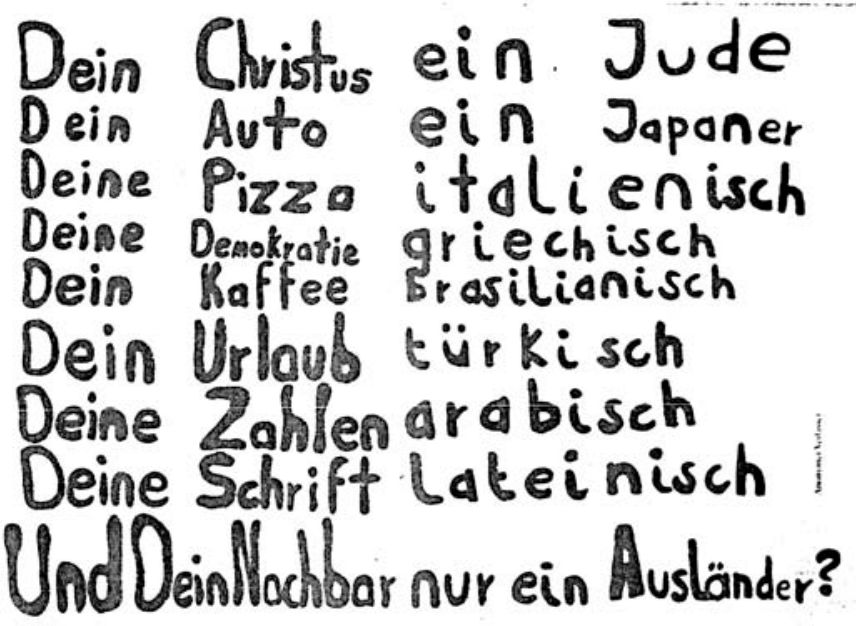

్ㅟ 
Regeln lassen sich im Sinne der Lernerprinzipien oft erstaunlich einfach darstellen und erklären (hier das Serialisierungsprinzip in Schritte international 1, 2005). Hieraus entsteht übrigens die Grundmotivation für didaktisierte Grammatiken.

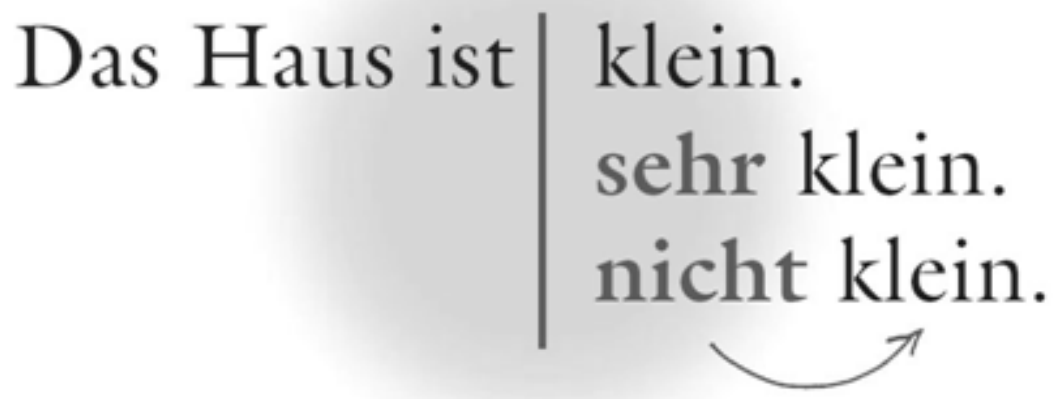

Animationen, wie dieses Beispiel aus uni-deutsch.de (www.deutsch-uni.com), fungieren als Einstieg in die Regelerklärung und die Vertiefung der grammatischen Kenntnisse.

Aussage

Position 1 Position 2

Mein Name ist Iris Pfeil.

Ich bin Timo.

Ich komme aus Deutschland.

Sie sprechen gut Deutsch.

\section{W-Frage}

Position 1 Position 2

Wer ist das?

Wie heißen Sie?

Woher kommen Sie?

Was sprechen Sie?

\section{wiederholen}

Chunks können zu jeder Zeit vermittelt und genutzt werden, ohne dass die Grammatik erklärt werden muss. Lerner sind erstaunlich aufnahmebereit für sol- 
che Ketten, die ähnlich verarbeitet werden wie einzelne Wörter (Beispiel aus Schritte international 1, 2005).

\author{
Etwas bewerten: Das ist _. \\ Das ist aber schön. \\ Das Bad ist doch nicht kein. \\ Meine Wohnung ist nicht so schorn. \\ Aber sie ist billig. \\ Das Haus gefallt mir. \\ Die Frau ist verruckt.
}

\title{
9 Schluss
}

Es wäre sinnvoll im Sprachunterricht, das nachzuempfinden, was in natürlicher Kommunikation passiert. Das bedeutet eine bessere Abstimmung von Eingabe und Aufnahmemöglichkeit der Lerner. Wir benötigen damit eine andere Vorstellung von grammatischer Progression in unseren Lehrwerken, auch eine andere, als sie der Referenzrahmen vorschlägt. Spracherwerb ist nun mal ein Prozess, den man nicht beliebig steuern kann. Im Mittelpunkt stehen immer die Begriffe, die Inhalte. Die Grammatik kommt zu den Inhalten, zu den Begriffen, und nicht umgekehrt.

Sinnvolle Konsequenzen aus diesen Beobachtungen können daher sein:

1. Eine bessere Abstimmung von Strukturen der Eingabe und Aufnahmeund Verarbeitungsmöglichkeiten der Lerner.

2. Eine Änderung und Differenzierung im Verständnis von Progression. Diese müsste ausgerichtet sein am L2-Erwerb und den Verarbeitungsmöglichkeiten der Lerner, d. h., pragmatische Elemente sind in den Vordergrund zu rücken und es ist auf einem lexikalischen Ansatz aufzubauen (vgl. hierzu die CD-ROM grenzenlos, www.grenzenlos-life.de).

3. Die Progression müsste nach rezeptiven und produktiven Kriterien konsequent unterschieden werden. Der Input sollte demnach Varianten mit verschiedener Komplexität (reiche Eingabe) enthalten. Sprachmischungen könnten dabei durchaus auch eine Brückenrolle spielen. In der Produktion sollten von den Lernern zunächst nur solche authentischen Textsorten genutzt werden, die einfache Strukturen und Formeln enthalten, jedoch keine grammatisch falschen Äußerungen und Texte. Auf dieser Basis kann dann die zunehmende Grammatikalisierung erfolgen.

4. In didaktisierten Grammatiken müssten die auf linguistischer und erwerbslinguistischer Forschung basierenden Bausteine systematisch zur 
Verfügung gestellt, in Lehrwerken konsequent umgesetzt und mit diesen empirisch erprobt werden. Anders wird sich die verbreitete Frustration beim Erwerb und der Vermittlung sprachlicher Strukturen nicht nachhaltig beseitigen lassen.

\section{Literatur}

Handwerker, Brigitte: Chunks, Raster und Regeln: Vom Lexikon zur Grammatik in der Fremdsprachenvermittlung. In: Börner, Wolfgang; Vogel, Klaus (Hrsg.): Kognitive Linguistik und Fremdsprachenerwerb. Tübingen: Gunter Narr, 2002, 207-230.

Givòn, Talmy: »Fro Discourse to Syntax: Grammar as a Processing Stategy«. Discours and Syntax. In: Givòn, Talmy (Hrsg.): Syntax and Semantics. Volume 12, New York: Academic Press, 1979, 81-112.

Hinnenkamp, Volker: Foreigner Talk und Tarzanisch. Eine vergleichende Studie über die Sprechweise gegenüber Ausländern am Beispiel des Deutschen und des Türkischen. Hamburg: Buske, 1982.

Krashen, Stephan: The Input Hypothesis: Issues and Imlications. London, New York: Longman, 1985.

Roche, Jörg: Xenolekte. Struktur und Variation im Deutsch gegenüber Ausländern. Berlin, New York, de Gruyter, 1987.

Roche, Jörg: Variation in Xenolects. In: Ammon, Ulrich et al. (Hrsg.): Variationslinguistik. Tübingen, Niemeyer, 1998. 117-139.

Roche, Jörg: Interkulturelle Sprachdidaktik. Eine Einführung. Tübingen, Gunter Narr, 2001 (= narr studienbücher).

Roche, Jörg: Fremdsprachenerwerb - Fremdsprachendidaktik. Tübingen: Franke, 2005. 